\title{
Utilização de diferentes métodos para a determinação da idade óssea em jovens
}

http://dx.doi.org/10.11606/1807-5509201700040741

\author{
Vanessa Carla Monteiro PINTO* \\ Francisco Emílio Simplício de SOUZA* \\ Thaisys Blanc dos Santos SIMÕES* \\ Arnaldo Luis MORTATTI* \\ Paulo Moreira Silva DANTAS* \\ Breno Guilherme de Araújo Tinôco CABRAL*
}

${ }^{*}$ Centro de

Ciências da Saúde

Universidade Federal

do Rio Grande do

Norte, Natal, RN,

Brasil.

\section{Resumo}

0 estudo tem como objetivo correlacionar os resultados da idade óssea em meninos e meninas em diferentes métodos. Na metodologia para verificar a idade óssea, foram realizadas aferições de estatura, diâmetros de úmero e fêmur, perímetro corrigido de braço, dobra tricipital, idade cronológica para compor o modelo matemático, e raio-x de mão e punho para utilização do método de Grave e Brown (1976). No tratamento estatístico realizado no programa SPSS 20.0 utilizando estatística descritiva, correlação de Pearson e diferença significativa entre os métodos. As correlações obtiveram resultados positivos para meninos e meninas, respectivamente, com resultados entre raio $x$ e idade cronológica $(M=0,84 / F=0,53)$, modelo matemático $(M=0,85 / F=0,56)$ e estatura $(M=0,80 / F=0,28)$, assim como entre o modelo matemático e estatura $(M=0,95 /$ $\mathrm{F}=0,84)$ e idade cronológica $(\mathrm{M}=0,95 / \mathrm{F}=0,76)$. Concluímos, desta forma, existir correlação entre os métodos, não existindo diferença significativa entre os mesmos, destacando assim o modelo matemático por ser um método prático e de fácil aplicação para uso no meio esportivo.

PalavRAS-Chave: Morfologia; Desenvolvimento Humano; Esportes.

\section{Introdução}

A ciência do esporte investiga fenômenos do desenvolvimento humano oriundos de três funções básicas dos seres vivos: crescimento, desenvolvimento e maturação. Este último, sendo muito complexo, requer uma confluência multidisciplinar para obter e desenvolver um entendimento compreensível ${ }^{1}$.

Dessa forma, a maturação é vista como um processo biológico contínuo e dinâmico, que se inicia na concepção e termina na morte. É pontuado por alteraçóes visíveis na estatura, composiçáo corporal e características sexuais secundárias, que culminam na transição da fase pré-reprodutiva para a reprodutiva do ciclo de vida humano ${ }^{2}$.

A maturação esquelética é amplamente reconhecida como o melhor indicador isolado do estado de maturidade, sendo considerada como um registro verdadeiro da idade biológica. Todas as crianças iniciam com um esqueleto de cartilagem e progridem até um esqueleto totalmente ossificado, adulto. No caso dos ossos tubulares (ossos longos e curtos), a maturidade é atingida quando ocorre a completa fusão das epífises com suas diáfises correspondentes; no caso dos ossos com formato irregular, a maturidade é definida pela morfologia adulta ${ }^{3}$.

O método mais utilizado de avaliaçáo da maturidade esquelética é o de Grave e Brown, o qual observa a sequência de eventos da ossificação através do raio $\mathrm{x}$ utilizando o atlas proposto por Pyle, Waterhouse e Greulich ${ }^{4}$, tal atlas, auxilia a determinar a maturidade óssea, através da procura da figura da radiografia correspondente ao sexo e idade cronológica do indivíduo que estamos estudando e verificar nas radiografias adjacentes (mais velha e mais nova) qual dos três filmes mais se assemelha superficialmente à radiografia a ser avaliada. Outra técnica bastante utilizada e o qual se utiliza do método de estimativa é a técnica por Tanner-Whitehouse, divididas em três estágios: 
TW1, onde cada osso da mão e do punho era analisado separadamente, registrando apenas um dos 8 estágios possíveis. TW2, atribuição de valores de maturidade diferenciados para ossos carpais, rádio, ulna e ossos curtos, incluindo a diferenciação por sexo. Com isso, permite a estimativa de estatura adulta. E por fim, TW3 é o mais atual e apresenta maior confiabilidade dos métodos para avaliar a maturidade óssea, em que possibilita estimar, separadamente, a idade óssea do rádio, ulna e ossos curtos por escala RUS (13 ossos) ${ }^{5}$.

O desenvolvimento da uma equaçáo preditora de idade óssea é um marco na literatura, pois se trata de um método menos invasivo e menos oneroso, que possibilita ser aplicada no ambiente esportivo e escolar para analisar a maturidade de iniciantes na prática esportiva, utilizando apenas variáveis antropométricas, que permitem a observação do estágio maturidade do individuo ${ }^{6}$.

\section{Método}

\section{Populaçáo e amostra}

Estudo correlacional com tipologia transversal. A amostra composta por escolares pré-púberes e púberes de ambos os sexos, com idades entre 8 a 14 anos, totalizando vinte e cinco indivíduos $(\mathrm{n}=25)$, sendo 14 do sexo feminino e 11 do sexo masculino. A escolha ocorreu de forma não probabilística intencional.

\section{Procedimentos}

A participação das crianças e adolescentes foi condicionada a que os responsáveis assinassem o Termo de Consentimento Livre e Esclarecido (TCLE), após serem informados sobre os objetivos do presente estudo.

A seguir, foram mensuradas as seguintes variáveis antropométricas: estatura corporal, massa corporal, perímetro corrigido de braço, dobra tricipital, diâmetro biepicondilar do úmero e do fêmur, seguindo a padronização da Internacional Society for Advancement in Kinanthropometry $(\mathrm{ISAK})^{10}$.

A idade óssea foi verificada com realização do exame radiológico de máo e punho, mediante laudo médico. Foi administrada uma única dosagem de
A maturação óssea diferentemente da idade cronológica é uma avaliação que consegue justificar em que estágio de maturidade a criança e $o$ adolescente se encontram ${ }^{7,8}$. Jovens de diferentes estágios maturacionais são frequentemente encontrados em um mesmo grupo de treinamento ou categoria esportiva, o que pode favorecer aos mais adiantados no processo de desenvolvimento biológico, além de desmotivar outros mais tardios, ou seja, avaliar a idade cronológica de um indivíduo sem observar a idade biológica deixa a ideia de estar desperdiçando potenciais atletas 9 .

Nesse contexto, a busca por um método de identificação da maturação óssea mais econômico, prático e sem a necessidade de especialistas tem sido uma constante nas ciências do esporte ${ }^{6}$. Diante do exposto, o presente estudo tem como objetivo estabelecer uma correlaçáo entre os protocolos de verificação de idade óssea.

Raio-x, de forma individualizada. A análise do laudo foi feita por um mesmo avaliador com relevante experiência, através do método proposto por Grave e Brown descrito no trabalho de Pyle, Waterhouse, Greulich $^{4}$ para comparação e determinação da idade óssea sendo considerado na literatura como padrão ouro.

O modelo matemático preditor da maturação óssea utilizado foi o proposto por $\mathrm{CABRAL}^{6}$, no qual a idade óssea é determinada a partir das variáveis antropométricas conforme a equação, Idade Óssea $=-11,620+7,004$ (estatura) $+1,226$. Dsexo + 0,749 (idade) $-0,068(\mathrm{Tr})+0,214(\mathrm{Pcb})-0,588(\mathrm{Du})$ $+0,388$ (Df).

Onde: $\operatorname{Tr}=$ dobra cutânea tricipital; $\mathrm{Pcb}=$ perímetro do braço; $\mathrm{DU}=$ diâmetro ósseo do úmero; Df = diâmetro ósseo do fêmur; e Dsexo $=0$ para o sexo masculino e Dsexo $=1$, para o sexo feminino.

Os testes realizados atenderam rigorosamente aos protocolos escolhidos para observação das variáveis em questão, seguindo os procedimentos previamente autorizados pelo Comitê de ética responsável (parecer No071/071/2010), atendendo aos itens propostos pela resolução 466/12-CNS-Brasil, e respeitando as normas internacionais de experimentação com humanos. 


\section{Instrumentos}

Os instrumentos utilizados foram: balança eletrônica Filizola 110, com capacidade para $150 \mathrm{~kg}$ e divisóes de $1 / 10$ de $\mathrm{kg}$, a estatura foi obtida através um estadiômetro Sanny ${ }^{\circledR}$ ES2020, para a aferição das dobras cutâneas foi utilizado compasso científico Sanny ${ }^{\circ}$, para diâmetros ósseos paquímetro da marca Sanny ${ }^{\oplus}$ com precisão de $0,1 \mathrm{~cm}$ e fita antropométrica metálica da marca Sanny ${ }^{\circ}$ com precisão de $0,1 \mathrm{~mm}$ para o perímetro corrigido de braço. As radiografias foram realizadas por um aparelho da marca Rhos ${ }^{\oplus}$, com ampola Toshiba ${ }^{\oplus}$ de $60 \mathrm{Kvp}$ e $9 \mathrm{~mA}$, a uma distância foco-filme de $75 \mathrm{~cm}$. O filme utilizado foi Kodak ${ }^{\circledast}$ TMATX de 18 x $24 \mathrm{~cm}$, em chassi com écran de terras raras, revelados em uma processadora automática DENTXR, com componentes químicos para Kodak ${ }^{\oplus}$.

\section{Tratamento estatístico}

Para o tratamento estatístico das informações, foi utilizado o programa SPSS Versão 20.0. Na análise estatística, verificou-se o pressuposto de distribuição normal dos dados através do teste de Kolmogorov-Smirnov e, posteriormente, foram calculados os coeficientes de correlação de Pearson. $\mathrm{O}$ nível de significância para $\mathrm{p}<0,05$. Obtiveram-se os respectivos intervalos de confiança (IC de 95\%) e os valores foram ainda analisados pelos limites de concordância do gráfico de Bland e Altman, onde tomaram um valor alfa de $5 \%$.

\section{Resultados e discussão}

Os resultados abaixo não apresentaram diferenças significativas entre os métodos em meninas e meninos.

Na TABELA 2 é demonstrada a correlação dos protocolos e suas variáveis em meninos.

A idade óssea por raio-x de máo e punho quando confrontado com a variável de idade cronológica apresentou alta correção nos meninos.

Apesar de Malina e Bouchard ${ }^{11}$, que mencionarem que o crescimento e a maturação biológica nas crianças não ocorrem necessariamente em sincronia com a idade cronológica, em nosso estudo, verifica-se que a idade óssea por raio-x de mão e punho, quando confrontada com a variável de idade cronológica, apresentou alta correção nos meninos, fato que pode ser explicado ao se constatar que, apesar de poder haver distorçôes na sincronia das referidas variáveis, estatisticamente a diferença não deverá ser destacada quando se tratar de indivíduos sem distúrbios de desenvolvimento biológico.

TABELA 1 - Caracterização da amostra masculina e feminina.

\begin{tabular}{lccccc}
\hline & \multicolumn{2}{c}{ Meninos } & & \multicolumn{2}{c}{ Meninas } \\
\cline { 2 - 3 } \cline { 5 - 6 } & Média & Desvio & & Média & Desvio \\
\hline Estatura & 1,45 & 0,11 & & 1,62 & 0,04 \\
\hline Idade Cronologica & 10,58 & 1,49 & & 13,36 & 0,04 \\
\hline Raio X & 10,09 & 1,92 & & 14,14 & 1,28 \\
\hline Equação Preditora & 10,02 & 2,10 & & 14,88 & 1,24 \\
\hline
\end{tabular}

TABELA 2 - Correlações dos protocolos de idade óssea em meninos.

\begin{tabular}{lcccc}
\hline & Raio X & Modelo Matemático & Idade Cronológica & Estatura \\
\hline Raio-X & 1 & $0,848^{* *}$ & $0,839^{* *}$ & $0,803^{* *}$ \\
\hline Modelo Matemático & $0,848^{* *}$ & 1 & $0,948^{* *}$ & $0,947^{* *}$ \\
\hline Idade Cronológica & $0,839^{* *}$ & $0,948^{* *}$ & 1 & $0,947^{* *}$ \\
\hline
\end{tabular}

Neste estudo, fica evidenciado a influência da variável estatura sobre a idade óssea por raio-x de acordo com o alto índice de correlação nos meninos. $\mathrm{O}$ pico de velocidade de crescimento (PVC) ocorreu
A magnitude dessas correlações foi avaliada qualitativamente de acordo com Hopkins et al. (2009) (>0,70 - elevado, 0,30 a 0,70 moderado e $<0,30$ - fraco). 
de forma simultânea à idade óssea. Segundo MaLIna et al. ${ }^{12}$, à medida que há um avanço no processo maturacional da criança, a tendência é que, com as mudanças fisiológicas e metabólicas, o individuo atinja com maior velocidade a sua estatura final.

A variável estatura, quando analisada com o protocolo de idade cronológica em meninos, apresentou alta correlação. Nesse caso, o resultado observado no presente estudo revela consonância com os resultados observados por FraINer et al. ${ }^{13}$ e CABRAL ${ }^{6}$, em que a estatura e a idade cronológica expóem maior correlação entre as variáveis com a idade óssea, tais estudos evidenciam ainda a estatura como variável de grande importância no esporte de forma geral.

Os valores do raio-x de mão e punho confrontados com os valores estimados pelo modelo preditor apresentaram alta correlação em meninos, devido às variáveis componentes do modelo. $\mathrm{O}$ mesmo foi encontrado, quando o protocolo de raio-x foi analisado com a estatura, e isso se deve ao fato de que o tecido ósseo mineralizado é um tecido dinâmico, com potencial para crescer e remodelar-se, permanecendo ativo durante toda a vida ${ }^{14}$. O seu crescimento está relacionado aos centros de ossificação, a partir dos quais irá sofrer processos de maturação, até que o crescimento cesse com a fusão das epífises ${ }^{15}$.

Idade Cronológica relacionada com o modelo preditor apresentou alta correlação, onde, de acordo com a literatura, as mudanças ocorridas em cada individuo estáo diretamente ligadas aos estágios maturacionais, sendo este um fator de influência de forma crescente sobre os aspectos antropométricos, discutidos e confirmados nos estudos científicos ${ }^{16}$. Tais resultados podem ainda serem reforçados com os estudos de VeIGA ${ }^{17}$, que confirmam a alta correlação entre a idade óssea e a maturação analisando diferentes métodos, a partir da predição da idade relativa.

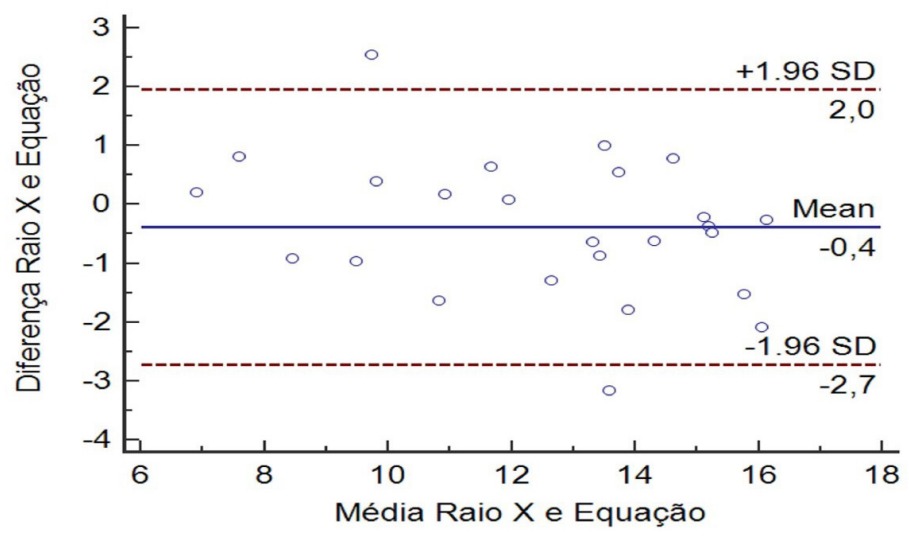

GRÁFICO 1 - Bland e Altman de Raio X e Equação Preditora.

No GRÁFICO 1, na análise do gráfico Bland e Altman, as dispersões mostram que em sua maioria as variáveis coincidem e se aproximam da linha central, o que revela bons níveis de coincidência entre os resultados observados para $\mathrm{IO}$ em raio $\mathrm{x}$ e na equação. Estudando amostras de crianças brasileiras, TaVANo et al. ${ }^{18}$ também encontraram coeficientes de correlação elevados entre as idades óssea e cronológica, assim como Cole et al. ${ }^{19}$ No estudo em questão, por se tratar de uma estatística que observa níveis de coincidência ou relação entre duas variáveis, optou-se por analisar os grupos simultaneamente, tendo os mesmos apresentado bons resultados de correlação.

TABELA 3 - Correlações dos protocolos de idade óssea em meninas.

Níveis de significância $>0,70$ : elevado; 0,30 a 0,70 : moderado; $<0,30$ : fraco.

\begin{tabular}{lcccc}
\hline & Raio-X & Modelo Matemático & Idade Cronológica & Estatura \\
\hline Raio-X & 1 & $0,533^{*}$ & $0,559^{*}$ & $0,283^{*}$ \\
\hline Modelo Matemático & $0,533^{*}$ & 1 & $0,759^{*}$ & $0,386^{*}$ \\
\hline Idade Cronológica & $0,599^{*}$ & $0,759^{*}$ & 1 & $0,836^{* *}$ \\
\hline
\end{tabular}


A TABELA 3 representa a correlação dos protocolos e variáveis em meninas. Nesta amostra, as variáveis observadas apresentaram fracas e moderadas correlaçôes com os protocolos.

Nas meninas, a amostra apresentou níveis de correlação moderado, quando confrontados os protocolos de raio-x com a variável de estatura, onde a literatura tem apontado que o pico de velocidade de crescimento em meninas acontece durante a puberdade, fato esse também observado em diferentes estudos que constatam que a maturação observada através da idade da menarca influencia diretamente nos fatores antropométricos das meninas ${ }^{20,21}$.

Foi observada ainda a correlaçáo entre o protocolo de raio-x e a variável da idade cronológica em meninas. Embora as participantes tenham faixa etária aproximada, apresentam início e ritmo de progressáo maturacional muito variáveis, isto é, o evento da puberdade náo necessariamente acontecem na mesma idade cronológica para diferentes ou mesmas populaçóes. Isto porque este evento pode ser influenciado por diversos fatores, principalmente por ocorrência de uma maturaçáo cada vez mais precoce no homem através do tempo ${ }^{22}$.

Analisando ainda a idade cronológica, relacionando-a com a variável da estatura, apresentou correlação moderada. Ocorrendo ou não um surto no pico de velocidade de crescimento, não sendo muito compatível com a idade cronológica.

Frente à diferença entre os gêneros, ao observar a idade óssea, os resultados encontrados corroboram os estudos que concluíram que todos os eventos do crescimento e fases do esqueleto aconteceram mais cedo em indivíduos do gênero feminino do que em indivíduos do gênero masculino ${ }^{23}$.

Com o presente estudo, fica evidenciada a positiva correlação entre os protocolos de raio-x de mão e punho e o modelo matemático preditor baseado em variáveis antropométricas, ambos para verificação de idade óssea, tendo resultados significativos em ambos os gêneros, com pequeno destaque para o gênero masculino, que apresentou melhores correlaçóes nesse estudo, evidenciando que estas foram menos significativas nas meninas.

Dessa forma, confirmamos o modelo matemático preditor de idade óssea como uma objetiva ferramenta de avaliação para professores de Educação Física e esporte, que poderão usufruir deste instrumento de fácil aplicabilidade, como método auxiliar no processo de orientação e seleção de talentos na iniciação esportiva.

\section{Abstract}

\section{Different bone age assessment methods for young people}

The study aims to analyze the results of bone age in boys and girls from different methods. In the methodology were performed measurements of height, diameter of the humerus and femur, corrected arm girth, triceps fold, chronological age to compose the mathematical model predictor of bone age, along with $x$-ray of hand and wrist. Statistical analysis was performed with SPSS 20.0 using descriptive statistics, Pearson correlation and significant difference among the methods. The correlations were positive for boys and girls respectively with results from $x$-ray and chronological age $(M=0,839 / F=0,533)$, mathematical model $(M=0,848 / F=0,559)$ and height $(M=0,803 / F=0,283)$ as well as between the mathematical model and height $(M=0,947 / F=0,836)$ and chronological age $(M=0,948 / F=0,759)$. We conclude that there is a correlation among the results of the methods, with no significant differences, thus highlighting the mathematical model since it is a practical and easy method to be used in sports.

KEYWORDS: Morphology; Human Development; Sports.

\section{Referências}

1. Mohamed H, Vaeyens R, Matthys S, Multael M, Lefevre J, Lenoir M, et al. Anthropometric and performance measures for the development of a talent detection and identification model in youth hand-ball. J Sports Sci. 2009;27(3):257-66. 
2. Cameron N. Assessment of maturation. In: Cameron N, Bogin B, editores. Human growth and development. San Diego: Academic Press; 2002. p. 363-82.

3. Bogin B. Patterns of human growth. 2a ed. Cambridge: Cambridge University Press; 1999.

4. Pyle SI, Waterhouse AM, Greulich WW. Attributes of the radiographic standard of reference for the National Health Examination Survey. Am J Phys Anthropol. 1971;35(3):331-7.

5. Oliveira Júnior A, Lopes G, Ramos D. Avaliação da maturação em crianças e jovens. Revista Hupe. 2013;12(4):38-46.

6. Cabral BGAT. Equação preditora de idade óssea na iniciação esportiva através de variáveis antropométricas. Rev Bras Med Esporte. 2013;19(2):99-103.

7. Beunen G, Malina RM. Growth and biological maturation: relevance to athletic performance. In: Bar-Or O, editor. The child and adolescent athlete encyclopaedia of sports medicine. New Jersey: Blackwell Science; 1996.

8. Malina RM, Bouchard C. Growth, maturation, and physical activity. Champaign: Human Kinetics; 1991.

9. Keizer SM, Mul D. Tendências no desenvolvimento puberal na Europa. Hum Reprod Update. 2001;7:287-91.

10. Marfell-Jones M, Olds T, Stewart A, Carter L. International standards for anthropometric assessment-ISAK. South Africa: Potchefstroom; 2006.

11. Malina RM, Bouchard C. Atividade física do atleta jovem: do crescimento à maturação. 1a ed. São Paulo: Roca; 2002.

12. Malina RM, Bouchard C, Bar-Or O. Growth, maturation, and physical activity. 2a ed. Champaign: Human Kinetics; 2004.

13. Frainer DES, Oliveira FR, Pazin J. Influência da maturação sexual, idade cronológica e índices de crescimento no limiar de lactato e no desempenho da corrida de 20 minutos. Rev Bras Med Esporte. 2006;12(3):139-44.

14. Uysal T, Sari Z, Ramoglu SI, Basciftci FA. Relationships between dental and skeletal maturity in Turkish subjects. Angle Orthod. 2004;74(5):657-64.

15. Katchburian E, Arana V. Histologia e embriologia oral. São Paulo: Médica Panamericana; 1999.

16. Little BB, Malina RM. Gene-environment interaction in skeletal maturity and body dimensions of urban Oaxaca Mestizo schoolchildren. Ann Hum Biol. 2006;34(2):216-25.

17. Veiga VWM. Maturação e performance de jovem andebolista [dissertação]. Lisboa: Universidade Técnica de Lisboa, Faculdade de Motricidade Humana; 2009.

18. Tavano O, Freitas JAS, Lopes ES. Greulich \& Pyle e Tanner \& Whitehouse: comparação entre duas tabelas de avaliação de idade biológica através do desenvolvimento ósseo. Clin Pediatr. 1982;5(6):7-21.

19. Cole AJL, Webb L, Cole TJ. Bone age estimation: a comparison of methods. Br J Radiol. 1988;61(728):683-6.

20. Bergmann GG. Crescimento somático aptidão física relacionada a saúde e estilo de vida de escolares de 10 a 14 anos: um estudo longitudinal [dissertação]. Porto Alegre: Universidade Federal do Rio Grande do Sul; 2006.

21. Roman EP, Ribeiro RR, Guerra Junior G, Barros Filho AA. Antropometry, sexual maturation and menarcheal age according to socioeconomic status of schoolgirls from Cascavel (PR). Rev Assoc Med Bras. 2009;55(3):317-21.

22. Damian MF, Woitchunas FE, Cericato GO, Cechinato F, Moro G, Massochin ME, et al. Análise da confiabilidade e da correlação de dois índices de estimativa da maturação esquelética: índice carpal e índice vertebral. Rev Dent Press Ortodon Ortop Facial. 2006; 11(5):110-20.

23. Hilgenberg S, Pinto SCS, Pinheiro JC, Jimenez EEO, Coelho U. Comparação entre as idades óssea, dentária e cronológica por meio de método radiográfico simplificado. Rev Odonto. 2008;16(32):31-8.

\begin{abstract}
ENDEREÇO
Vanessa Carla Monteiro Pinto

Rua Antônio Prado, 7 - Cidade da Esperança 59071-440 - Natal - RN - BRASIL e-mail: vanecmpinto@gmail.com; nessa_carla@hotmail.com
\end{abstract}

Recebido para publicação: 24/o3/2015

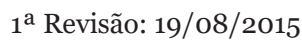

$2^{\text {a }}$ Revisão: $14 / 10 / 2015$

Aceito: 07/03/2016 